\title{
THE TRAJECTORY ATTRACTOR \\ AND ITS LIMITING BEHAVIOR \\ FOR THE CONVECTIVE BRINKMAN-FORCHHEIMER EQUATIONS
}

\author{
Caidi ZhaO - Lei Kong - Guowei Liu — Min ZhaO
}

\begin{abstract}
This paper studies the trajectory behavior of the convective Brinkman-Forchheimer equations in three-dimensional (3D) bounded domains. We first prove the existence of the trajectory attractor $\mathcal{A}_{\alpha}^{\mathrm{tr}}$ for the natural translation semigroup in the trajectory space. Then we establish that the trajectory attractor $\mathcal{A}_{\alpha}^{\text {tr }}$ converges, as $\alpha \rightarrow 0^{+}$, to the trajectory attractor $\mathcal{A}_{0}^{\text {tr }}$ of the $3 \mathrm{D}$ Navier-Stokes equations in a proper topological space.
\end{abstract}

\section{Introduction}

The three-dimensional (3D) convective Brinkman-Forchheimer (CBF) equations have the form (see e.g. [16])

$$
\begin{gathered}
\frac{\partial u}{\partial t}-\nu \Delta u+(u \cdot \nabla) u+\alpha u+\beta|u|^{r-1} u+\nabla p=g, \\
\nabla \cdot u=0, \quad x \in \Omega,
\end{gathered}
$$

2010 Mathematics Subject Classification. 35B40, 35Q30, 35G25.

Key words and phrases. Convective Brinkman-Forchheimer equations, trajectory attractor, Navier-Stokes equations, topological space.

This paper is sponsored by the National NSFC (No.11271290), the 973 Program with Grant No. 2012CB426510, the Graduate Students Innovation Fund of Wenzhou University (No. 31606036010181). 
where $u$ is the velocity field of the fluid, $g$ is the external force function and the scalar function $p$ is the pressure. $(u \cdot \nabla) u$ denotes the convective effect and $\nabla \cdot u=0$ describes the incompressibility of the fluid. In equation (1.1), $r>1$ is a constant, $\nu, \alpha$ and $\beta$ are parameters, where $\nu$ is the Brinkman coefficient (effective viscosity), $\alpha$ is the Darcy coefficient (viscosity divided by permeability), and $\beta$ is the Forchheimer coefficient. We assume that $\Omega \subset \mathbb{R}^{3}$ is an open bounded smooth domain in this paper.

When $\alpha=\beta=0$, equations (1.1)-(1.2) correspond to the classical 3D incompressible Navier-Stokes (NS for short) equations (see [24]). The general case $\alpha \beta \neq 0$ can be also considered as the so-called tamed NS equations, see [22]. Equations (1.1)-(1.2) without the convective effect (i.e. the term $(u \cdot \nabla) u$ disappears) are called the incompressible Brinkman-Forchheimer (BF for short) equations. The $\mathrm{BF}$ and $\mathrm{CBF}$ equations are used as a mathematical model to describe the motion of fluid flow in a saturated porous medium, see, e.g. [12], [14], [22], [23], [32]. This model is recognized to be more accurate when the flow velocity is too large for the Darcy's law to be valid alone and in addition the porosity is not too small.

There are some references studying the BF and CBF equations (see e.g. [16], [23], [30], [33], [34]). The structural stability in terms of the continuous dependence of solutions to the parameters $\nu, \alpha$ and $\beta$ of the CBF equations has been investigated by some authors, see [11], [13], [20], [21] and the references therein. The asymptotic behavior of solutions for the autonomous BF equations has been studied by [16], [19], [26], [30]. In [26], the existence of a global attractor in $\mathbb{H}^{1}(\Omega)$ norm was proved for the external force $g \in \mathbb{L}^{2}(\Omega)$ being time-invariant. In [30] the existence of a global attractor in $\mathbb{H}^{2}(\Omega)$ norm was proved with a new and effective approach. Very recently, by conducting maximal regularity estimates, Kalantarov and Zelik (see [16]) proved the existence of the smooth attractor for the autonomous $\mathrm{BF}$ and $\mathrm{CBF}$ equations with nonlinearities of arbitrary higherorder growth rate.

In the present paper, we discuss the following $\mathrm{CBF}$ equations

$$
\begin{gathered}
\frac{\partial u}{\partial t}-\nu \Delta u+(u \cdot \nabla) u+\alpha u+\alpha|u|^{r-1} u+\nabla p=g, \quad t>0, \\
\nabla \cdot u=0,
\end{gathered}
$$

with the initial and boundary value conditions

$$
\begin{aligned}
\left.u\right|_{\partial \Omega} & =0, \\
u(x, 0) & =u_{0} .
\end{aligned}
$$

Here we take $\alpha=\beta>0$ in equation (1.3). We assume that $r \in(1,3]$ in the whole paper, and in this case we can obtain the existence of weak solutions to equations (1.3)-(1.6) corresponding to each initial value. However, it is not known whether 
weak solutions are unique or not. The reason is that one can guarantee $(u \cdot \nabla) u \in$ $L^{4 / 3} \subset L^{q}$ with the assumption $r \geq 3$, where $q=(1+r) / r$, and therefore, in contrast to the case of the classical NS equations, the multiplication of (1.3) by $u$ with integration over $\Omega$ is justified for any weak solution of that equation (see Kalantarov and Zelik [16]). The possible non-uniqueness of the weak solutions is one of the difficult problems to overcome when we discuss the asymptotic behavior of weak solutions for this equations.

There are three notions introduced to overcome the difficulties associated to possible non-uniqueness of solutions in the study of dynamical systems generated from partial differential equations. The first one is generalized semi-flow which was formulated by Ball [2]. The second one is multi-valued dynamical systems, one can refer to Melnik and Valero [17], [18] for multi-valued semi-flows, Caraballo et al. [9], [10], Kapustyan and Valero [15], Wang and Zhou [31] for multi-valued process or semiprocess, and Caraballo et al. [6], [7], [8] for multivalued random dynamical systems. The third one is trajectory attractor. The definition of trajectory attractor was initially developed to overcome difficulties related to possible non-uniqueness of weak solutions for the 3D NS equations (see e.g. [3], [4], [27]). Later, the theory of trajectory attractor has been proved very useful for other models whose solution corresponding to each initial state can be non-unique.

The first purpose of the present paper is to use the third notion mentioned above to prove the existence of trajectory attractor $\mathcal{A}_{\alpha}^{\text {tr }}$ for equations (1.3)-(1.6). To this end, we first construct the trajectory space $\mathcal{T}_{\alpha}^{+}$of solutions and consider the natural translation semigroup $\{S(t)\}_{t \geq 0}$ acting on it. Then we prove that $\{S(t)\}_{t \geq 0}$ possesses an absorbing set $\Lambda \subset \mathcal{T}_{\alpha}^{+}$, which is bounded in the Banach space $\mathcal{F}_{\alpha,+}^{b}$ and is compact in the topology $\Theta_{\alpha,+}^{\text {loc }}$ (see the notations in Section 3).

Compared with the CBF equations discussed in [16], this paper takes $r \in$ $(1,3]$, while article [16] needs $r>3$ to guarantee the uniqueness of the weak solutions for the CBF equations. In fact, the nonlinear term $\alpha|u|^{r-1} u$ leads to an additional difficulty in deriving the estimates of solutions under the acting of $\{S(t)\}_{t \geq 0}$ in the trajectory space. Also this nonlinear term will cause a small difficulty when we verify the closedness of the trajectory space in the topology $\Theta_{\alpha,+}^{\text {loc }}$ (see Lemma 3.6). These facts require from us to do more detailed analysis to deal with this nonlinear term.

The second aim of this paper is to verify the convergence of the trajectory attractor $\mathcal{A}_{\alpha}^{\text {tr }}$ to the trajectory attractor $\mathcal{A}_{0}^{\text {tr }}$ of the $3 \mathrm{D}$ incompressible NS equations as $\alpha \rightarrow 0^{+}$. When $\alpha=0$, equations (1.3)-(1.4) turn into the classical $3 \mathrm{D}$ incompressible NS equations. It is well-known (see e.g. [4, 28]) that the 3D incompressible NS equations possess a trajectory attractor (denoted by) $\mathcal{A}_{0}^{\text {tr }}$ in its trajectory space (denoted by) $\mathcal{T}_{0}^{+}$. We shall first prove that the solutions of 
the CBF equations converge to $\mathcal{A}_{0}^{\text {tr }}$ and then $\mathcal{A}_{\alpha}^{\text {tr }} \rightarrow \mathcal{A}_{0}^{\text {tr }}$ in the topology $\Theta_{+}^{\text {loc }}$ as $\alpha \rightarrow 0^{+}$.

The rest of the paper is organized as follows. In Section 2 we first introduce some notations and operators. Then we show the existence and recall some properties of the weak solutions for the CBF equations. In Section 3, we prove the existence of the trajectory attractor $\mathcal{A}_{\alpha}^{\text {tr }}$ for the CBF equations. In Section 4 , we verify the convergence of the weak solutions and the trajectory attractor $\mathcal{A}_{\alpha}^{\text {tr }}$ to the trajectory attractor $\mathcal{A}_{0}^{\text {tr }}$ of the 3D incompressible NS equations as $\alpha \rightarrow 0^{+}$.

\section{Preliminaries}

In this section, we first introduce some notations and put the initial boundary value problem (1.3)-(1.6) into the abstract form. Then we show the existence of the weak solutions and recall two useful lemmas.

In this paper we use $c$ to denote the generic constant that can take different values in different places. $\mathbb{L}^{p}(\Omega)=L^{p}(\Omega) \times L^{p}(\Omega) \times L^{p}(\Omega)$ is the 3D Lebesgue space with norm $\|\cdot\|_{\mathbb{L}^{p}(\Omega)}, \mathbb{H}_{0}^{m}(\Omega)=H_{0}^{m}(\Omega) \times H_{0}^{m}(\Omega) \times H_{0}^{m}(\Omega)$ is the $3 \mathrm{D}$ Sobolev space with norm $\|\cdot\|_{\mathbb{H}_{0}^{m}(\Omega)}$ and dual space $\mathbb{H}^{-m}(\Omega)$, where $L^{p}(\Omega)$ and $H_{0}^{m}(\Omega)$ are the usual $L^{p}$-Lebesgue space and Sobolev space ([1]) on $\Omega$, respectively. When $p=2$, we denote $\|\cdot\|_{\mathbb{L}^{2}(\Omega)}=\|\cdot\|$, and also $\|\cdot\|_{L^{2}(\Omega)}=\|\cdot\|$ if there is no confusion. We define

$$
\mathcal{V}=\left\{\phi \mid \phi \in\left(\mathcal{C}_{0}^{\infty}(\Omega)\right)^{3} \text { and } \nabla \cdot \phi=0\right\},
$$

and denote by $H, \widetilde{\mathbb{L}}^{p}(\Omega)$ and $V$ the closure spaces of $\mathcal{V}$ in $\mathbb{L}^{2}$ norm, $\mathbb{L}^{p}(\Omega)$ norm and $\mathbb{H}_{0}^{1}$ norm, respectively. $\langle\cdot, \cdot\rangle$ is the inner product of $H$ (or $\mathbb{L}^{2}(\Omega), L^{2}(\Omega)$ ), or the dual pairing between $V$ and $V^{\prime}$ (the dual space of $V$ ), or between $\mathbb{H}_{0}^{m}(\Omega)$ and $\mathbb{H}^{-m}(\Omega)$.

To put equations (1.3)-(1.6) into the abstract form, we now introduce some operators. Firstly, set $\mathcal{L}=-\Delta$ (the Laplace operator $\Delta$ is taken with zero boundary conditions $\left.\left.u\right|_{\partial \Omega}\right)$. Then using integration by parts, we have

$$
\langle\mathcal{L} u, v\rangle=\langle\nabla u, \nabla v\rangle, \quad \text { for all } u, v \in \mathbb{H}_{0}^{1}(\Omega) \text { or } V .
$$

Secondly, we define a continuous trilinear form (see e.g. [25]) $b(\cdot, \cdot, \cdot)$ on $\mathbb{H}_{0}^{1}(\Omega)$ (and in particular on $V$ ) by

$$
b(u, v, w)=\sum_{j, k=1}^{3} \int_{\Omega} u_{j} \frac{\partial v_{k}}{\partial x_{j}} w_{k} d x, \quad \text { for all } u, v, w \in \mathbb{H}_{0}^{1}(\Omega) \text { or } V .
$$

If $u \in V$, one can check

$$
b(u, v, w)=-b(u, w, v), \quad b(u, v, v)=0, \quad \text { for all } v, w \in \mathbb{H}_{0}^{1}(\Omega) .
$$


For $u, v \in \mathbb{H}_{0}^{1}(\Omega)$ (resp. $V$ ), we denote by $B(u, v)$ the element in $\mathbb{H}^{-1}(\Omega)$ (resp. $V^{\prime}$ ) defined by $\langle B(u, v), w\rangle=b(u, v, w)$, for any $w \in \mathbb{H}_{0}^{1}(\Omega)$ (resp. $V$ ). Set

$$
B(u)=B(u, u), \quad \text { for all } u \in \mathbb{H}_{0}^{1}(\Omega) \text { (resp. } V \text { ). }
$$

Excluding the pressure $p$, we can rewrite the weak form of problem (1.3)-(1.6) in the solenoidal vector fields as follows:

$$
\begin{gathered}
\frac{\partial u}{\partial t}+\nu \mathcal{L} u+B(u)+\alpha u+\alpha|u|^{r-1} u=g, \quad \text { in } \mathcal{D}^{\prime}\left(0, T ; V^{\prime}\right), \\
u(x, 0)=u_{0} .
\end{gathered}
$$

Definition 2.1. A function $u \in L^{\infty}(0, T ; H) \cap L^{2}(0, T ; V) \cap L^{r+1}(0, T$; $\left.\widetilde{\mathbb{L}}^{r+1}(\Omega)\right)$ is called a weak solution of equation $(2.5)$ on the interval $(0, T)$ if $u$ together with its derivative $\partial_{t} u$ satisfies equation (2.5) in the sense of distribution in $\mathcal{D}^{\prime}\left(0, T ; V^{\prime}\right)$.

Lemma 2.2 ([16]). Let $\alpha>0, r \in(1,3]$ and $g \in V^{\prime}$. Then for each $T>0$ and for any $u_{0} \in H$, equations (2.5)-(2.6) possess at least one weak solution which satisfies the following energy inequality

$$
\frac{1}{2} \frac{d}{d t}\|u(t)\|^{2}+\nu\|u(t)\|_{V}^{2}+\alpha\left(\|u(t)\|^{2}+\|u(t)\|_{\widetilde{\mathbb{L}}^{r+1}(\Omega)}^{r+1}\right) \leq\langle u(t), g\rangle,
$$

for all $t \in(0, T)$, in the sense that

$$
\begin{aligned}
& -\frac{1}{2} \int_{0}^{T}\|u(s)\|^{2} \psi^{\prime}(s) d s+\nu \int_{0}^{T}\|u(s)\|_{V}^{2} \psi(s) d s \\
& \quad+\alpha \int_{0}^{T}\left(\|u(s)\|^{2}+\|u(s)\|_{\widetilde{\mathbb{L}}^{r+1}(\Omega)}^{r+1}\right) \psi(s) d s \leq \int_{0}^{T}\langle u(s), g\rangle \psi(s) d s,
\end{aligned}
$$

for all $\psi(s) \in \mathcal{C}_{0}^{\infty}(0, T), \psi(s) \geq 0$.

We end this section with two useful lemmas.

Lemma 2.3 ([4]). Let $E_{1}$ be a Banach space and $E \hookrightarrow E_{0} \subseteq E_{1}$, where the embedding $E \hookrightarrow E_{0}$ is compact. Set

$$
W_{p, q}\left(0, T ; E, E_{1}\right)=\left\{\phi(t), t \in[0, T] \mid \phi(t) \in L^{p}(0, T ; E), \phi^{\prime}(t) \in L^{q}\left(0, T ; E_{1}\right)\right\},
$$

$p \geq 1, q>1$, with norm

$$
\|\phi\|_{W_{p, q}}=\left(\int_{0}^{T}\|\phi\|_{E}^{p}\right)^{1 / p}+\left(\int_{0}^{T}\left\|\phi^{\prime}\right\|_{E_{1}}^{q}\right)^{1 / q} .
$$

Then $W_{p, q}\left(0, T ; E, E_{1}\right) \hookrightarrow L^{p}\left(0, T ; E_{0}\right)$ with compact embedding.

Lemma $2.4([4])$. Let $y(s), K(s) \in L_{\mathrm{loc}}^{1}(0,+\infty)$ and let

$$
\text { (2.9) }-\int_{0}^{+\infty} y(s) \phi^{\prime}(s) d s+\beta \int_{0}^{+\infty} y(s) \phi(s) d s \leq \int_{0}^{+\infty} K(s) \phi(s) d s
$$


hold for any $\phi(s) \in \mathcal{C}_{0}^{\infty}\left(\mathbb{R}_{+}\right)$with $\phi(s) \geq 0$ and some $\beta \in \mathbb{R}$. Then, for any $t, \tau \in \mathbb{R}_{+}, t \geq \tau$, there holds

$$
y(t) \mathrm{e}^{\beta t}-y(\tau) \mathrm{e}^{\beta \tau} \leq \int_{\tau}^{t} K(s) \mathrm{e}^{\beta s} d s .
$$

\section{Existence of the trajectory attractor for the CBF equations}

In this section, we will prove the existence of the trajectory attractor for the $\mathrm{CBF}$ equation (2.5). In the sequel, we use $\Pi_{+}$to denote the restriction operator (with respect to (w.r.t.) the time variable) to the semi-infinite interval $\mathbb{R}_{+}$. Analogously, $\Pi_{T}$ stands for the restriction operator to the interval $[0, T]$. For example, if $u(\cdot) \in L^{\infty}\left(\mathbb{R}_{+}, H\right) \cap L_{\text {loc }}^{2}\left(\mathbb{R}_{+}, V\right)$ then $\Pi_{T} u(\cdot) \in L^{\infty}(0, T ; H) \cap$ $L^{2}(0, T ; V)$, and $\Pi_{T} u(t)=u(t)$ if $t \in[0, T]$.

When we prove Lemma 2.2, the Galerkin method makes it possible to construct a family of weak solutions of equation (2.5) that satisfy the energy inequality (2.7) in the sense of (2.8). These weak solutions constitute the trajectory space of equation (2.5).

Definition 3.1. The trajectory space $\mathcal{T}_{\alpha}^{+}$of equation (2.5) consists of functions $u \in L_{\text {loc }}^{\infty}\left(\mathbb{R}_{+} ; H\right) \cap L_{\text {loc }}^{2}\left(\mathbb{R}_{+} ; V\right) \cap L_{\text {loc }}^{r+1}\left(\mathbb{R}_{+} ; \widetilde{\mathbb{L}}^{r+1}(\Omega)\right)$ such that for all $T>0$ the function $\Pi_{T} u$ is a weak solution of equation (2.5) on the interval $(0, T)$ and $\Pi_{T} u$ satisfies the energy inequality (2.7) in the sense of (2.8).

We consider the natural translation semigroup $\{S(t)\}_{t \geq 0}$ acting on the trajectory space $\mathcal{T}_{\alpha}^{+}$. The operator $S(t)$ is defined as

$$
S(t) u(\cdot)=u(t+\cdot), \quad u(\cdot) \in \mathcal{T}_{\alpha}^{+}, \quad \text { for all } t \geq 0 .
$$

Lemma 3.2. (a) For any $u_{0} \in H$, there exists a trajectory (maybe not unique) $u \in \mathcal{T}_{\alpha}^{+}$such that $u(0)=u_{0}$;

(b) $\mathcal{T}_{\alpha}^{+}$is translation invariant under the acting of $\{S(t)\}_{t \geq 0}$, i.e.

$$
S(t) \mathcal{T}_{\alpha}^{+} \subset \mathcal{T}_{\alpha}^{+}, \quad \text { for all } t \geq 0 .
$$

Proof. Assertion (a) follows directly from Lemma 2.2. We next prove (b). Let a function $u(s) \in \mathcal{T}_{\alpha}^{+}$with $s \in \mathbb{R}_{+}$. Since equation (2.5) is autonomous, the function $S(t) u(s)=u(t+s), t \geq 0, s \geq 0$, is clearly a weak solution of equation (2.5), and $u(t+s)$ meets the energy inequality (2.7), as does the function $u(s)$.

LEMma 3.3. If $u \in L^{2}(0, T ; V) \cap L^{\infty}(0, T ; H) \cap L^{r+1}\left(0, T ; \widetilde{\mathbb{L}}^{r+1}(\Omega)\right)$, then the functions

$$
\begin{aligned}
t & \mapsto \mathcal{L} u(t) \in L^{2}\left(0, T ; V^{\prime}\right), \\
t & \mapsto B(u(t)) \in L^{4 / 3}\left(0, T ; V^{\prime}\right), \\
t & \mapsto \alpha|u(t)|^{r-1} u(t) \in L^{(r+1)^{*}}\left(0, T ; \widetilde{\mathbb{L}}^{(r+1)^{*}}(\Omega)\right),
\end{aligned}
$$

where $(r+1)^{*}=(r+1) / r$ is the conjugated exponent of $r+1$. 
Proof. For almost every $t \in[0, T], \mathcal{L} u(t)$ and $B(u(t))$ are elements of $V^{\prime}$, and $\alpha|u(t)|^{r-1} u(t)$ is an element of $\widetilde{\mathbb{L}}^{(r+1)^{*}}(\Omega)$. The measurability of the functions $t \mapsto \mathcal{L} u(t), t \mapsto B(u(t))$ and $t \mapsto \alpha|u(t)|^{r-1} u(t)$ is not difficult to check. Now for any $\phi \in V$, we have

$$
|\langle\mathcal{L} u, \phi\rangle|=|\langle\nabla u, \nabla \phi\rangle| \leq c\|u\|_{V}\|\phi\|_{V} .
$$

By Hölder and Gagliardo-Nirenberg inequalities and the embedding $V \hookrightarrow \widetilde{\mathbb{L}}^{6}(\Omega)$, we have (see e.g. [28])

$$
|\langle B(u), \phi\rangle| \leq c\|u\|^{1 / 2}\|u\|_{V}^{3 / 2}\|\phi\|_{V},
$$

and

$$
\begin{aligned}
\alpha\left|\left\langle|u|^{r-1} u, \phi\right\rangle\right| & \leq \alpha \int_{\Omega}|u|^{r}|\phi| d x \leq \alpha\left\||u|^{r}\right\|_{\widetilde{\mathbb{L}}^{(r+1)^{*}}(\Omega)}\|\phi\|_{\mathbb{L}^{(r+1)}(\Omega)} \\
& =\alpha\|u\|_{\widetilde{\mathbb{L}}^{(r+1)}(\Omega)}^{r}\|\phi\|_{\mathbb{L}^{(r+1)}(\Omega)} .
\end{aligned}
$$

(3.6)-(3.8) imply that

$$
\begin{aligned}
\|\mathcal{L} u(t)\|_{V^{\prime}} & \leq c\|u(t)\|_{V}, \\
\|B(u(t))\|_{V^{\prime}} & \leq c\|u(t)\|^{1 / 2}\|u(t)\|_{V}^{3 / 2}, \\
\left\|\alpha|u(t)|^{r-1} u(t)\right\|_{\widetilde{\mathbb{L}}^{(r+1)^{*}}(\Omega)} & \leq c\|u(t)\|_{\widetilde{\mathbb{L}}^{r+1}(\Omega)}^{r} .
\end{aligned}
$$

Therefore,

$$
\begin{aligned}
\int_{0}^{T}\|\mathcal{L} u(t)\|_{V^{\prime}}^{2} d t & \leq c \int_{0}^{T}\|u(t)\|_{V}^{2} d t \\
\int_{0}^{T}\|B(u(t))\|_{V^{\prime}}^{4 / 3} d t & \leq c \int_{0}^{T}\|u(t)\|^{2 / 3}\|u(t)\|_{V}^{2} d t \\
& \leq c\|u(t)\|_{L^{\infty}(0, T ; H)}^{2 / 3} \int_{0}^{T}\|u(t)\|_{V}^{2} d t, \\
\int_{0}^{T}\left\|\alpha|u(t)|^{r-1} u(t)\right\|_{\widetilde{\mathbb{L}}^{(r+1)^{*}(\Omega)}}^{(r+1)^{*}} d t & \leq c \int_{0}^{T}\|u(t)\|_{\widetilde{\mathbb{L}}^{r+1}(\Omega)}^{r+1} d t .
\end{aligned}
$$

(3.12)-(3.14) imply that (3.3)-(3.5) hold true. The proof is complete.

We now introduce the following spaces

$$
\begin{aligned}
\mathcal{F}_{\alpha,+}^{\mathrm{loc}}:= & L_{\mathrm{loc}}^{\infty}\left(\mathbb{R}_{+} ; H\right) \cap L_{\mathrm{loc}}^{2}\left(\mathbb{R}_{+} ; V\right) \cap L_{\mathrm{loc}}^{r+1}\left(\mathbb{R}_{+} ; \widetilde{\mathbb{L}}^{r+1}(\Omega)\right) \\
& \cap\left\{u(\cdot) \mid \partial_{t} u(\cdot) \in L_{\mathrm{loc}}^{4 / 3}\left(\mathbb{R}_{+} ; V^{\prime}\right)\right\}, \\
\Pi_{T} \mathcal{F}_{\alpha,+}^{\mathrm{loc}}:= & L^{\infty}(0, T ; H) \cap L^{2}(0, T ; V) \cap L^{r+1}\left(0, T ; \widetilde{\mathbb{L}}^{r+1}(\Omega)\right) \\
& \cap\left\{u(\cdot) \mid \partial_{t} u(\cdot) \in L^{4 / 3}\left(0, T ; V^{\prime}\right)\right\} .
\end{aligned}
$$


The topology in $\Pi_{T} \mathcal{F}_{\alpha,+}^{\text {loc }}$ is defined as the following weak convergence: let $\left\{u_{n}(x, t)\right\}$ be a sequence of $\Pi_{T} \mathcal{F}_{\alpha,+}^{\text {loc }}$, if

$$
\begin{cases}u_{n}(x, t) \rightarrow u(x, t) & \text { weak* in } L^{\infty}(0, T ; H) \\ u_{n}(x, t) \rightarrow u(x, t) & \text { weakly in } L^{2}(0, T ; V) \\ u_{n}(x, t) \rightarrow u(x, t) & \text { weakly in } L^{r+1}\left(0, T ; \widetilde{\mathbb{L}}^{r+1}(\Omega)\right) \\ \partial_{t} u_{n}(x, t) \rightarrow \partial_{t} u(x, t) & \text { weakly in } L^{4 / 3}\left(0, T ; V^{\prime}\right)\end{cases}
$$

then we say that the sequence of functions $\left\{u_{n}(x, t)\right\}$ converges to $u(x, t)$ in the topology of $\Pi_{T} \mathcal{F}_{\alpha,+}^{\text {loc }}$ as $n \rightarrow \infty$. The topology of space $\mathcal{F}_{\alpha,+}^{\text {loc }}$ is the inductive limit of the topologies in the spaces $\Pi_{T} \mathcal{F}_{\alpha,+}^{\text {loc }}$, i.e. a sequence of functions $\left\{u_{n}(x, t)\right\} \subset \mathcal{F}_{\alpha,+}^{\text {loc }}$ converges to $u(x, t) \in \mathcal{F}_{\alpha,+}^{\text {loc }}$ in the topology of $\mathcal{F}_{\alpha,+}^{\text {loc }}$ as $n \rightarrow \infty$ if $\Pi_{T} u_{n}(x, t) \longrightarrow \Pi_{T} u(x, t)$ in the topology of $\Pi_{T} \mathcal{F}_{\alpha,+}^{\text {loc }}$ for any $T>0$. We denote by $\Theta_{\alpha,+}^{\text {loc }}$ the space $\mathcal{F}_{\alpha,+}^{\text {loc }}$ with this topology. We also define a Banach space $\mathcal{F}_{\alpha,+}^{b}$ as

$$
\mathcal{F}_{\alpha,+}^{b}:=\left\{u(x, t) \in \mathcal{F}_{\alpha,+}^{\mathrm{loc}} \mid\|u\|_{\mathcal{F}_{\alpha,+}^{\mathrm{b}}}<+\infty\right\},
$$

where the norm in $\mathcal{F}_{\alpha,+}^{b}$ is defined as

$$
\begin{aligned}
\|u\|_{\mathcal{F}_{\alpha,+}^{b}}:= & \sup _{t \geq 0}\left\{\|S(t) u\|_{L^{\infty}(0,1 ; H)}+\|S(t) u\|_{L^{2}(0,1 ; V)}\right. \\
& \left.+\|S(t) u\|_{L^{r+1}\left(0,1 ; \widetilde{\mathbb{L}}^{r+1}(\Omega)\right)}+\left\|S(t) \partial_{t} u\right\|_{L^{4 / 3}\left(0,1 ; V^{\prime}\right)}\right\}
\end{aligned}
$$

Here we want to point out that the spaces $\Theta_{\alpha,+}^{\text {loc }}$ and $\mathcal{F}_{\alpha,+}^{b}$ introduced above are modifications of the spaces $\Theta_{+}^{\text {loc }}$ and $\mathcal{F}_{+}^{b}$ introduced in [4].

Lemma 3.4. $\mathcal{T}_{\alpha}^{+} \subset \mathcal{F}_{\alpha,+}^{\text {loc }}$.

Proof. Since $r \in(1,3]$ and $\widetilde{\mathbb{L}}^{(r+1)^{*}}(\Omega) \hookrightarrow V^{\prime}$, we have by (3.5) that

$$
\alpha|u(t)|^{r-1} u(t) \in L_{\text {loc }}^{(r+1)^{*}}\left(\mathbb{R}_{+} ; \widetilde{\mathbb{L}}^{(r+1)^{*}}(\Omega)\right) \hookrightarrow L_{\text {loc }}^{4 / 3}\left(\mathbb{R}_{+} ; V^{\prime}\right) .
$$

By Lemma 3.3, we get from equation $(2.5)$ that $\partial_{t} u(\cdot) \in L_{\text {loc }}^{4 / 3}\left(\mathbb{R}_{+} ; V^{\prime}\right)$.

Lemma 3.5. $\mathcal{T}_{\alpha}^{+} \subset \mathcal{F}_{\alpha,+}^{b}$ and for any trajectory $u \in \mathcal{T}_{\alpha}^{+}$, there exist some positive constants $c_{0}, \rho_{0}, \eta_{0}$ and $\delta_{0}$, which are independent of $u$, such that

$$
\|S(t) u\|_{\mathcal{F}_{\alpha,+}^{\mathrm{b}}} \leq c_{0}\|u\|_{L^{\infty}(0,1 ; H)}^{\eta_{0}} \mathrm{e}^{-\delta_{0} t}+\rho_{0}, \quad \text { for all } t \geq 0
$$

where $\eta_{0} \in\{1 / 2,1,3 / 2,2,5 / 2,3\}, \delta_{0} \in\{\alpha / 2,3 \alpha / 2, \alpha / 4,3 \alpha / 4,9 \alpha / 4, \alpha\}$.

Proof. By (2.8), we have for any $\psi(s) \in \mathcal{C}_{0}^{\infty}\left(\mathbb{R}_{+}\right)$with $\psi(s) \geq 0$ that

$$
-\int_{0}^{+\infty}\|u\|^{2} \psi^{\prime}(s) d s+\alpha \int_{0}^{+\infty}\|u\|^{2} \psi(s) d s \leq \frac{1}{\nu} \int_{0}^{+\infty}\|g\|_{V^{\prime}}^{2} \psi(s) d s .
$$

Using Lemma 2.4, we obtain

$$
\|u(t)\|^{2} \mathrm{e}^{\alpha t} \leq\|u(\tau)\|^{2} \mathrm{e}^{\alpha \tau}+\frac{\|g\|_{V^{\prime}}^{2}}{\alpha \nu}\left(\mathrm{e}^{\alpha t}-\mathrm{e}^{\alpha \tau}\right), \quad \text { for all } t, \tau \in \mathbb{R}_{+} \text {with } t \geq \tau,
$$


from which we get

(3.21) $\quad\|S(t) u\|_{L^{\infty}\left(\mathbb{R}_{+} ; H\right)} \leq c\|u\|_{L^{\infty}(0,1 ; H)} \mathrm{e}^{-\alpha t / 2}+\frac{\|g\|_{V^{\prime}}}{\sqrt{\alpha \nu}}, \quad$ for all $t \in \mathbb{R}_{+}$.

Now, using $u$ to take dual paring $\langle\cdot, \cdot\rangle$ with equation (2.5) and integrating with respect to time variable from $\tau$ to $t\left(t, \tau \in \mathbb{R}_{+}\right.$with $\left.t \geq \tau\right)$, we obtain

$$
\begin{gathered}
\frac{1}{2}\left(\|u(t)\|^{2}-\|u(\tau)\|^{2}\right)+\nu \int_{\tau}^{t}\|u\|_{V}^{2} d s+\alpha \int_{\tau}^{t}\|u\|^{2} d s+\alpha \int_{\tau}^{t}\|u\|_{\widetilde{\mathbb{L}}^{r+1}}^{r+1} d s \\
\leq \int_{\tau}^{t}\|g\|_{V^{\prime}}\|u(s)\|_{V} d s \leq \frac{1}{2 \nu} \int_{\tau}^{t}\|g\|_{V^{\prime}}^{2} d s+\frac{\nu}{2} \int_{\tau}^{t}\|u(s)\|_{V}^{2} d s
\end{gathered}
$$

which implies

$\|u(t)\|^{2}+\nu \int_{\tau}^{t}\|u\|_{V}^{2} d s \leq \frac{1}{\nu} \int_{\tau}^{t}\|g\|_{V^{\prime}}^{2} d s+\|u(\tau)\|^{2}, \quad$ for all $t, \tau \in \mathbb{R}_{+}$with $t \geq \tau$.

We use (3.21) to get

$$
\begin{aligned}
\|S(t) u\|_{L^{2}(0,1 ; V)} & =\left(\int_{t}^{t+1}\|u(s)\|_{V}^{2} d s\right)^{1 / 2} \\
& \leq\left(\frac{\|u(t)\|^{2}}{\nu}+\frac{\|g\|_{V^{\prime}}^{2}}{\nu^{2}}\right)^{1 / 2} \leq \frac{\|u(t)\|}{\sqrt{\nu}}+\frac{\|g\|_{V^{\prime}}}{\nu} \\
& \leq c\|u\|_{L^{\infty}(0,1 ; H)} \mathrm{e}^{-\alpha t / 2}+\frac{\|g\|_{V^{\prime}}}{2 \nu \sqrt{\alpha}}+\frac{\|g\|_{V^{\prime}}}{\nu}
\end{aligned}
$$

for all $t \geq 0$. Combining (3.21)-(3.23), we have

$$
\begin{aligned}
\|S(t) u\|_{L^{r+1}\left(0,1 ; \widetilde{\mathbb{L}}^{r+1}(\Omega)\right)} & =\left(\int_{t}^{t+1}\|u(s)\|_{\widetilde{\mathbb{L}}^{r+1}(\Omega)}^{r+1} d s\right)^{1 / r+1} \\
& \leq\left(\frac{1}{2 \alpha \nu}\|g\|_{V^{\prime}}^{2}+\frac{1}{2 \alpha}\|u(t)\|^{2}\right)^{1 / r+1} \\
& \leq c\left(\|u\|_{L^{\infty}(0,1 ; H)}^{2} \mathrm{e}^{-\alpha t}+\|g\|_{V^{\prime}}^{2}\right)^{1 / r+1} .
\end{aligned}
$$

Since $r \in(1,3]$, we get from (3.24) that

(3.25) $\|S(t) u\|_{L^{r+1}\left(0,1 ; \widetilde{\mathbb{L}}^{r+1}(\Omega)\right)}$

$$
\begin{aligned}
& \leq c\left(\|u\|_{L^{\infty}(0,1 ; H)}^{2} \mathrm{e}^{-\alpha t}+\|g\|_{V^{\prime}}^{2}\right)^{1 / 2}+c\left(\|u\|_{L^{\infty}(0,1 ; H)}^{2} \mathrm{e}^{-\alpha t}+\|g\|_{V^{\prime}}^{2}\right)^{1 / 4} \\
& \leq c\left(\|u\|_{L^{\infty}(0,1 ; H)} \mathrm{e}^{-\alpha t / 2}+\|g\|_{V^{\prime}}\right)+c\left(\|u\|_{L^{\infty}(0,1 ; H)}^{1 / 2} \mathrm{e}^{-\alpha t / 4}+\|g\|_{V^{\prime}}^{1 / 2}\right) .
\end{aligned}
$$

Setting $\rho=(1 /(2 \nu \sqrt{\alpha})+1 / \nu)\|g\|_{V^{\prime}}$ and using (3.10) and (3.23), we get

$$
\begin{aligned}
& \left(\int_{t}^{t+1}\|B(u(s))\|_{V^{\prime}}^{4 / 3} d s\right)^{3 / 4} \leq c\left(\int_{t}^{t+1}\|u(s)\|^{2 / 3}\|u(s)\|_{V}^{2} d s\right)^{3 / 4} \\
& \quad \leq c \operatorname{esssup}_{t \geq 0}\|u(t)\|^{1 / 2}\left(\int_{t}^{t+1}\|u(s)\|_{V}^{2} d s\right)^{3 / 4} \\
& \quad \leq c\left(\|u\|_{L^{\infty}(0,1 ; H)} \mathrm{e}^{-\alpha t / 2}+\rho\right)^{1 / 2}\left(\|u\|_{L^{\infty}(0,1 ; H)} \mathrm{e}^{-\alpha t / 2}+\rho\right)^{3 / 2}
\end{aligned}
$$




$$
\begin{aligned}
& =c\left(\|u\|_{L^{\infty}(0,1 ; H)} \mathrm{e}^{-\alpha t / 2}+\rho\right)^{2} \\
& =c\|u\|_{L^{\infty}(0,1 ; H)}^{2} \mathrm{e}^{-\alpha t}+2 c \rho\|u\|_{L^{\infty}(0,1 ; H)} \mathrm{e}^{-\alpha t / 2}+c \rho^{2},
\end{aligned}
$$

for all $t \geq 0$. Now, from equation (2.5), we have

$$
\begin{aligned}
\left\|S(t) \partial_{t} u\right\|_{L^{4 / 3}\left(0,1 ; V^{\prime}\right)} \leq & \nu\|S(t) \mathcal{L} u\|_{L^{4 / 3}\left(0,1 ; V^{\prime}\right)} \\
& +\|S(t) B(u)\|_{L^{4 / 3}\left(0,1 ; V^{\prime}\right)}+\alpha\|S(t) u\|_{L^{4 / 3}\left(0,1 ; V^{\prime}\right)} \\
& +\alpha\left\|S(t)|u|^{r-1} u\right\|_{L^{4 / 3}\left(0,1 ; V^{\prime}\right)}+\|g\|_{L^{4 / 3}\left(0,1 ; V^{\prime}\right)}
\end{aligned}
$$

By (3.9) and the embedding $L^{2}(0,1 ; V) \hookrightarrow L^{4 / 3}(0,1 ; V)$, we get

$$
\|S(t) \mathcal{L} u\|_{L^{4 / 3}\left(0,1 ; V^{\prime}\right)} \leq c\|S(t) u\|_{L^{4 / 3}(0,1 ; V)} \leq c\|S(t) u\|_{L^{2}(0,1 ; V)} .
$$

Also by the embedding $L^{2}(0,1 ; V) \hookrightarrow L^{4 / 3}(0,1 ; V) \hookrightarrow L^{4 / 3}\left(0,1 ; V^{\prime}\right)$, we have

$$
\|S(t) u\|_{L^{4 / 3}\left(0,1 ; V^{\prime}\right)} \leq c\|S(t) u\|_{L^{2}(0,1 ; V)} .
$$

Since $(r+1)^{*}=(r+1) / r \geq 4 / 3$ when $r \in(1,3]$, we have $\widetilde{\mathbb{L}}^{(r+1)^{*}}(\Omega) \hookrightarrow V^{\prime}$ and $L^{(r+1)^{*}}\left(0,1 ; V^{\prime}\right) \hookrightarrow L^{4 / 3}\left(0,1 ; V^{\prime}\right)$. Thus

$$
\begin{array}{rl}
\| S(t)|u|^{r-1} & u\left\|_{L^{4 / 3}\left(0,1 ; V^{\prime}\right)} \leq c\right\| S(t)|u|^{r-1} u \|_{L^{(r+1)^{*}}\left(0,1 ; V^{\prime}\right)} \\
& \leq c\left\|S(t)|u|^{r-1} u\right\|_{L^{(r+1)^{*}}\left(0,1 ; \widetilde{\mathbb{L}}^{(r+1)^{*}}(\Omega)\right)} \\
& =c\|S(t) u\|_{L^{r+1}\left(0,1 ; \widetilde{\mathbb{L}}^{r+1}(\Omega)\right)}^{r} \\
& \leq c\|S(t) u\|_{L^{r+1}\left(0,1 ; \widetilde{\mathbb{L}}^{r+1}(\Omega)\right)}+c\|S(t) u\|_{L^{r+1}\left(0,1 ; \widetilde{\mathbb{L}}^{r+1}(\Omega)\right) .}^{3}
\end{array}
$$

Taking (3.21), (3.23) and (3.25)-(3.30) into account, we get (3.20).

We next use the estimation obtained in Lemma 3.5 to construct the absorbing set for $\{S(t)\}_{t \geq 0}$ in $\mathcal{T}_{\alpha}^{+}$.

Lemma 3.6. There is a bounded absorbing set $\Lambda \subset \mathcal{T}_{\alpha}^{+}$for $\{S(t)\}_{t \geq 0}$ in $\mathcal{T}_{\alpha}^{+}$, i.e. for any bounded (in the norm of $\mathcal{F}_{\alpha,+}^{b}$ ) subset $\mathcal{B} \subset \mathcal{T}_{\alpha}^{+}$, there exists a time $t_{0}=t_{0}(\mathcal{B})$ such that $S(t) u \in \Lambda$, for all $u \in \mathcal{B}$, for all $t \geq t_{0}$.

Proof. Set

$$
\Lambda=\left\{u \in \mathcal{T}_{\alpha}^{+} \mid\|u\|_{\mathcal{F}_{\alpha,+}^{b}} \leq 2 \rho_{0}\right\},
$$

where $\rho_{0}$ is the positive constant from Lemma 3.5. Then by (3.20), $\Lambda$ is obviously an absorbing set for $\{S(t)\}_{t \geq 0}$ in $\mathcal{T}_{\alpha}^{+}$.

Lemma 3.7. $\mathcal{T}_{\alpha}^{+}$is closed in the topology $\Theta_{\alpha,+}^{\mathrm{loc}}$.

Proof. Let $\left\{u_{n}\right\}$ be a bounded (in the norm of $\mathcal{F}_{\alpha,+}^{b}$ ) sequence in $\mathcal{T}_{\alpha}^{+}$and there exists a function $u^{*} \in \mathcal{F}_{\alpha,+}^{\text {loc }}$ such that

$$
u_{n} \rightarrow u^{*} \text { in the topology } \Theta_{\alpha,+}^{\text {loc }} \text { as } n \rightarrow \infty .
$$

We shall prove in two steps that $u^{*} \in \mathcal{T}_{\alpha}^{+}$. 
Step 1. We prove for each $T>0$ that $\Pi_{T} u^{*}$ is a weak solution of equation $(2.5)$ on $(0, T)$. To this end, we establish that $u^{*} \in L_{\text {loc }}^{\infty}\left(\mathbb{R}_{+} ; H\right) \cap L_{\text {loc }}^{2}\left(\mathbb{R}_{+} ; V\right) \cap$ $L_{\text {loc }}^{r+1}\left(\mathbb{R}_{+}, \widetilde{\mathbb{L}}^{r+1}(\Omega)\right)$, and $\forall T>0, \Pi_{T} u^{*}(t)$ is a weak solution of equation (2.5) on the interval $(0, T)$.

Indeed, since $\left\{u_{n}\right\} \subset \mathcal{T}_{\alpha}^{+}$and $\left\{u_{n}\right\}$ is bounded in the $\mathcal{F}_{\alpha,+}^{b}$ norm, we see for any $T>0$ that $\left\{\Pi_{T} u_{n}\right\}$ is bounded in $L^{\infty}(0, T ; H) \cap L^{2}(0, T ; V) \cap$ $L^{r+1}\left(0, T ; \widetilde{\mathbb{L}}^{r+1}(\Omega)\right)$ and $\left\{\Pi_{T} \partial_{t} u_{n}\right\}$ is bounded in $L^{4 / 3}\left(0, T ; V^{\prime}\right)$. Using the diagonal procedure we deduce that there exists a function $u \in L_{\text {loc }}^{\infty}\left(\mathbb{R}_{+} ; H\right) \cap$ $L_{\text {loc }}^{2}\left(\mathbb{R}_{+} ; V\right) \cap L_{\text {loc }}^{r+1}\left(\mathbb{R}_{+}, \widetilde{\mathbb{L}}^{r+1}(\Omega)\right)$ and a subsequence $\left\{u_{n_{k}}\right\}$ of $\left\{u_{n}\right\}$ such that

$$
\begin{aligned}
\Pi_{T} u_{n_{k}} & \rightarrow \Pi_{T} u & & \text { weakly in } L^{2}(0, T ; V) \text { as } n_{k} \rightarrow \infty, \\
u_{n_{k}} & \rightarrow u & & \text { weak* in } L^{\infty}(0, T ; H) \text { as } n_{k} \rightarrow \infty, \\
\Pi_{T} u_{n_{k}} & \rightarrow \Pi_{T} u & & \text { weakly in } L^{r+1}\left(0, T ; \widetilde{\mathbb{L}}^{r+1}(\Omega)\right) \text { as } n_{k} \rightarrow \infty, \\
\Pi_{T} \partial_{t} u_{n_{k}} & \rightarrow \Pi_{T} \partial_{t} u & & \text { weakly in } L^{4 / 3}\left(0, T ; V^{\prime}\right) \text { as } n_{k} \rightarrow \infty,
\end{aligned}
$$

for every $T>0$. Obviously, $\partial_{t} u \in L_{\text {loc }}^{4 / 3}\left(\mathbb{R}_{+} ; V^{\prime}\right)$. Thus $u_{n_{k}} \rightarrow u$ in the topology $\Theta_{\alpha,+}^{\text {loc }}$ as $n_{k} \rightarrow \infty$. From (3.32) and the uniqueness of limit we have $u=u^{*}$. Next we verify that $\Pi_{T} u^{*}$ is a weak solution of (2.5) on the interval $(0, T)$. To this end, we prove the following convergent relations:

$$
\begin{aligned}
(3.37) & \mathcal{L} \Pi_{T} u_{n_{k}} & \rightarrow \mathcal{L} \Pi_{T} u^{*} & & \text { weakly in } L^{2}\left(0, T ; V^{\prime}\right) \text { as } n_{k} \rightarrow \infty, \\
(3.38) & B\left(\Pi_{T} u_{n_{k}}\right) & \rightarrow B\left(\Pi_{T} u^{*}\right) & & \text { weakly in } L^{4 / 3}\left(0, T ; V^{\prime}\right) \text { as } n_{k} \rightarrow \infty, \\
(3.39) & \Pi_{T} u_{n_{k}} & \rightarrow \Pi_{T} u^{*} & & \text { weakly in } L^{2}\left(0, T ; V^{\prime}\right) \text { as } n_{k} \rightarrow \infty, \\
(3.40) & \Pi_{T}\left|u_{n_{k}}\right|^{r-1} u_{n_{k}} & \rightarrow \Pi_{T}\left|u^{*}\right|^{r-1} u^{*} & & \text { weakly in } L^{4 / 3}\left(0, T ; V^{\prime}\right) \text { as } n_{k} \rightarrow \infty .
\end{aligned}
$$

In fact, for all $\phi \in L^{2}(0, T ; V) \cap \mathcal{C}([0, T] ; V)$. We use (3.33) and the definition of the operator $\mathcal{L}$ to get

$$
\begin{aligned}
\lim _{n_{k} \rightarrow \infty} \int_{0}^{T}\left\langle\mathcal{L} \Pi_{T} u_{n_{k}}-\mathcal{L} \Pi_{T} u^{*}\right. & , \phi\rangle d t \\
& =\lim _{n_{k} \rightarrow \infty} \int_{0}^{T}\left\langle\Pi_{T} u_{n_{k}}-\Pi_{T} u^{*}, \mathcal{L} \phi\right\rangle d t=0,
\end{aligned}
$$

which implies that (3.37) holds true. The proof of (3.38) is the same as that in [27] where the existence of trajectory attractor for 3D NS equations was obtained. (3.39) is directly obtained from (3.33). We next prove (3.40). Let $\phi \in L^{4}(0, T ; V) \hookrightarrow L^{r+1}\left(0, T ; \widetilde{\mathbb{L}}^{r+1}(\Omega)\right)$. By (3.35) we have

$$
\Pi_{T}\left|u_{n_{k}}\right|^{r-1} u_{n_{k}} \rightarrow \Pi_{T}\left|u^{*}\right|^{r-1} u^{*} \quad \text { weakly in } L^{(r+1)^{*}}\left(0, T ; \widetilde{\mathbb{L}}^{(r+1)^{*}}(\Omega)\right) .
$$

Hence

$$
\lim _{n_{k} \rightarrow \infty} \int_{0}^{T}\left\langle\Pi_{T}\left|u_{n_{k}}\right|^{r-1} u_{n_{k}}-\Pi_{T}\left|u^{*}\right|^{r-1} u^{*}, \phi\right\rangle d t=0 .
$$


So (3.40) holds true. By (3.36)-(3.40) we can pass to the limit as $n_{k} \rightarrow \infty$. This shows that $\Pi_{T} u^{*}$ is a weak solution of equation $(2.5)$ on $(0, T)$.

Step 2. We prove that $\Pi_{T} u^{*}$ meets the energy inequality (2.7). Since $\left\{u_{n}\right\}$ is bounded in $\mathcal{F}_{\alpha,+}^{b}$ norm, there is some constant $C_{0}>0$ such that

$$
\left\|u_{n}\right\|_{\mathcal{F}_{\alpha,+}^{b}} \leq C_{0}, \quad \text { for all } n \in \mathbb{N},
$$

which implies that $\left\{\Pi_{T} u_{n}\right\}$ is bounded in $L^{2}(0, T ; V)$ and $\left\{\Pi_{T} \partial_{t} u_{n}\right\}$ is bounded in $L^{4 / 3}\left(0, T ; V^{\prime}\right)$. Note that $V \hookrightarrow H \hookrightarrow V^{\prime}$ with compact embeddings. It then follows from Lemma 2.3 and condition (3.32) that

$$
\Pi_{T} u_{n}(t) \rightarrow \Pi_{T} u^{*}(t) \quad \text { strongly in } L^{2}(0, T ; H) \text { as } n \rightarrow \infty .
$$

Thus, extracting a subsequence if necessary,

$$
\left\|\Pi_{T} u_{n}(t)\right\|^{2} \rightarrow\left\|\Pi_{T} u^{*}(t)\right\|^{2} \quad \text { for a.e. } T>0 \text { as } n \rightarrow \infty, t \in[0, T] .
$$

Now for any $\psi(s) \in \mathcal{C}_{0}^{\infty}(0, T)$ with $\psi(s) \geq 0$, the sequences of functions $\left\{\left\|\Pi_{T} u_{n}(t)\right\|^{2} \psi^{\prime}(t)\right\}$ and $\left\{\left\|\Pi_{T} u_{n}(t)\right\|^{2} \psi(t)\right\}$ belong to $L^{1}(0, T)$. From (3.42) we deduce that $\left\{\left\|\Pi_{T} u_{n}(t)\right\|^{2} \psi^{\prime}(t)\right\}$ and $\left\{\left\|\Pi_{T} u_{n}(t)\right\|^{2} \psi(t)\right\}$ are essentially bounded and thus possess an integrable majorant, respectively. Then by Lebesgue's dominated convergence theorem and (3.43)-(3.44), we get

$$
\begin{aligned}
& \lim _{n \rightarrow \infty} \int_{0}^{T}\left\|\Pi_{T} u_{n}(s)\right\|^{2} \psi^{\prime}(s) d s=\int_{0}^{T}\left\|\Pi_{T} u^{*}(s)\right\|^{2} \psi^{\prime}(s) d s, \\
& \lim _{n \rightarrow \infty} \int_{0}^{T}\left\|\Pi_{T} u_{n}(s)\right\|^{2} \psi(s) d s=\int_{0}^{T}\left\|\Pi_{T} u^{*}(s)\right\|^{2} \psi(s) d s .
\end{aligned}
$$

From (3.32) and (3.33), we have $\Pi_{T} u_{n}(\cdot) \sqrt{\psi(\cdot)} \rightarrow \Pi_{T} u^{*}(\cdot) \sqrt{\psi(\cdot)}$ weakly in $L^{2}(0, T ; V)$ and by the lower semicontinuity of norm that

$$
\int_{0}^{T}\left\|\Pi_{T} u^{*}(s)\right\|_{V}^{2} \psi(s) d s \leq \liminf _{n \rightarrow \infty} \int_{0}^{T}\left\|\Pi_{T} u_{n}(s)\right\|_{V}^{2} \psi(s) d s .
$$

Observing (3.43), we have

$$
\lim _{n \rightarrow \infty} \int_{0}^{T}\left\langle\Pi_{T} u_{n}(s), g\right\rangle \psi(s) d s=\int_{0}^{T}\left\langle\Pi_{T} u^{*}(s), g\right\rangle \psi(s) d s .
$$

Lastly, by (3.35) and also by the lower semicontinuity of norm

$$
\int_{0}^{T}\left\|\Pi_{T} u^{*}(s)\right\|_{\widetilde{\mathbb{L}}^{r+1}(\Omega)}^{r+1} \psi(s) d s \leq \liminf _{n \rightarrow \infty} \int_{0}^{T}\left\|\Pi_{T} u_{n}(s)\right\|_{\widetilde{\mathbb{L}}^{r+1}(\Omega)}^{r+1} \psi(s) d s .
$$

Since $u_{n}(t) \in \mathcal{T}_{\alpha}^{+}, n \in \mathbb{N}$, there holds

$$
\begin{aligned}
& (3.50) \quad-\frac{1}{2} \int_{0}^{T}\left\|\Pi_{T} u_{n}(s)\right\|^{2} \psi^{\prime}(s) d s+\nu \int_{0}^{T}\left\|\Pi_{T} u_{n}(s)\right\|_{V}^{2} \psi(s) d s \\
& +\alpha \int_{0}^{T}\left(\left\|\Pi_{T} u_{n}(s)\right\|^{2}+\left\|\Pi_{T} u_{n}(s)\right\|_{\widetilde{\mathbb{L}}^{r+1}(\Omega)}^{r+1}\right) \psi(s) d s \leq \int_{0}^{T}\left\langle\Pi_{T} u_{n}(s), g\right\rangle \psi(s) d s .
\end{aligned}
$$


Combining (3.45)-(3.49) and passing the limit in (3.50), we have

$$
\begin{gathered}
-\frac{1}{2} \int_{0}^{T}\left\|\Pi_{T} u^{*}(s)\right\|^{2} \psi^{\prime}(s) d s+\nu \int_{0}^{T}\left\|\Pi_{T} u^{*}(s)\right\|_{V}^{2} \psi(s) d s \\
+\alpha \int_{0}^{T}\left(\left\|\Pi_{T} u^{*}(s)\right\|^{2}+\left\|\Pi_{T} u^{*}(s)\right\|_{\widetilde{\mathbb{L}}^{r+1}(\Omega)}^{r+1}\right) \psi(s) d s \\
\leq \int_{0}^{T}\left\langle\Pi_{T} u^{*}(s), g\right\rangle \psi(s) d s .
\end{gathered}
$$

Combining steps one and two, we get $u^{*} \in \mathcal{T}_{\alpha}^{+}$.

Lemma 3.8. The absorbing set $\Lambda$ constructed by Lemma 3.6 is compact in the topology $\Theta_{\alpha,+}^{\text {loc }}$.

Proof. Let $\left\{u_{n}\right\} \subset \Lambda$ be a bounded (in the norm of $\mathcal{F}_{\alpha,+}^{b}$ ) sequence. Using the diagonal procedure we see that there exists a function $u \in \mathcal{F}_{\alpha,+}^{\text {loc }}$ and a subsequence $\left\{u_{n_{k}}\right\}$ of $\left\{u_{n}\right\}$ such that $u_{n_{k}} \longrightarrow u$ in the topology of $\Theta_{\alpha,+}^{\text {loc }}$. By Lemma 3.7, we have $u \in \mathcal{T}_{\alpha}^{+}$. At the same time, we obtain by the lower semi-continuity of norm that

$$
\|u\|_{\mathcal{F}_{\alpha,+}^{b}} \leq\left\|u_{n_{k}}\right\|_{\mathcal{F}_{\alpha,+}^{b}} \leq 2 \rho_{0}, \quad \text { for all } t \geq 0 .
$$

Therefore, $u \in \Lambda$. This proves the compactness of the absorbing set $\Lambda$ in the topology $\Theta_{\alpha,+}^{\text {loc }}$.

We now state and prove the main result of this section.

Theorem 3.9. Let $\alpha>0, r \in(1,3]$ and $g \in V^{\prime}$. Then the translation semigroup $\{S(t)\}_{t \geq 0}$ defined by (3.1) possesses a trajectory attractor $\mathcal{A}_{\alpha}^{\operatorname{tr}} \subset \mathcal{T}_{\alpha}^{+}$ with respect to the topology $\Theta_{\alpha,+}^{\text {loc }}$, which satisfies

(a) $\mathcal{A}_{\alpha}^{\text {tr }}$ is bounded in $\mathcal{F}_{\alpha,+}^{b}$ norm and is compact in the topology $\Theta_{\alpha,+}^{\text {loc }}$;

(b) $S(t) \mathcal{A}_{\alpha}^{\text {tr }}=\mathcal{A}_{\alpha}^{\text {tr }}$, for all $t \geq 0$;

(c) $\mathcal{A}_{\alpha}^{\text {tr }}$ is an attracting set for $\{S(t)\}_{t \geq 0}$ in the topology $\Theta_{\alpha,+}^{\text {loc }}$, i.e. for any $\mathcal{B} \subset \mathcal{T}_{\alpha}^{+}$bounded in $\mathcal{F}_{\alpha,+}^{b}$ and for any neighbourhood $\mathcal{O}\left(\mathcal{A}_{\alpha}^{\mathrm{tr}}\right)$ of $\mathcal{A}_{\alpha}^{\mathrm{tr}}$, there is a time $t^{*}=t^{*}(\mathcal{B}, \mathcal{O})$ such that $S(t) \mathcal{B} \subset \mathcal{O}\left(\mathcal{A}_{\alpha}^{\text {tr }}\right)$, for all $t \geq t^{*}$.

Proof. The result of this theorem follows from Lemmas 3.2(b), 3.4, 3.6, 3.8 and Theorem 4.1 of [28].

\section{Convergence of the trajectory attractor to that of the 3D NS equations}

In this section, we verify that the trajectory attractor $\mathcal{A}_{\alpha}^{\text {tr }}$ converges to the trajectory attractor $\mathcal{A}_{0}^{\text {tr }}$ of the 3D NS equations as $\alpha \rightarrow 0^{+}$. The convergence of solutions and trajectory attractors of the NS- $\alpha$ model and Leray- $\alpha$ model to those of the 3D NS equations were investigated in [5], [29]. 
4.1. Preliminary results on the $3 \mathrm{D}$ NS equations. Firstly, we recall some results concerning the following 3D incompressible NS equations (see e.g. [27]):

$$
\begin{aligned}
\frac{\partial u}{\partial t}-\nu \Delta u+(u \cdot \nabla) u+\nabla p & =g, \quad t>0, \\
\nabla \cdot u & =0, \\
\left.u\right|_{\partial \Omega} & =0, \\
u(x, 0) & =u_{0} .
\end{aligned}
$$

By excluding the pressure $p$, we can rewrite the weak form of problem (4.1)-(4.4) in the solenoidal vector fields as follows.

$$
\begin{aligned}
\frac{\partial u}{\partial t}+\nu \mathcal{L} u+B(u) & =g, \quad \text { in } \mathcal{D}^{\prime}\left(0, T ; V^{\prime}\right), \\
u(x, 0) & =u_{0} .
\end{aligned}
$$

Definition 4.1. A function $u \in L^{\infty}(0, T ; H) \cap L^{2}(0, T ; V)$ is called a weak solution of $(4.5)$ on the interval $(0, T)$ if $u$ together with its derivative $\partial_{t} u$ satisfies (4.5) in the sense of distribution in $\mathcal{D}^{\prime}\left(0, T ; V^{\prime}\right)$.

Lemma 4.2 ([4], [27]). Let $g \in V^{\prime}$. Then for each $T>0$ and for any $u_{0} \in H$, equations (4.5)-(4.6) possess at least one weak solution which satisfies the following energy inequality

$$
\frac{1}{2} \frac{\mathrm{d}}{d t}\|u(t)\|^{2}+\nu\|u(t)\|_{V}^{2} \leq\langle u(t), g\rangle, \quad \text { for all } t \in(0, T)
$$

in the sense that, for all $\psi(s) \in \mathcal{C}_{0}^{\infty}(0, T), \psi(s) \geq 0$,

$$
-\frac{1}{2} \int_{0}^{T}\|u(s)\|^{2} \psi^{\prime}(s) d s+\nu \int_{0}^{T}\|u(s)\|_{V}^{2} \psi(s) d s \leq \int_{0}^{T}\langle u(s), g\rangle \psi(s) d s .
$$

Definition $4.3([4],[27])$. The trajectory space $\mathcal{T}_{0}^{+}$of equation (4.5) consists of functions $u \in L_{\text {loc }}^{\infty}\left(\mathbb{R}_{+} ; H\right) \cap L_{\text {loc }}^{2}\left(\mathbb{R}_{+} ; V\right)$ such that for all $T>0$ the function $\Pi_{T} u$ is a weak solution of equation (4.5) on the interval $(0, T)$ and $\Pi_{T} u$ satisfies the energy inequality (4.7) in the sense of (4.8).

Write

$$
\begin{aligned}
&(4.9) \quad \mathcal{F}_{+}^{\text {loc }}:=L_{\mathrm{loc}}^{\infty}\left(\mathbb{R}_{+} ; H\right) \cap L_{\mathrm{loc}}^{2}\left(\mathbb{R}_{+} ; V\right) \cap\left\{u(\cdot) \mid \partial_{t} u(\cdot) \in L_{\mathrm{loc}}^{4 / 3}\left(\mathbb{R}_{+} ; V^{\prime}\right)\right\}, \\
& \text { (4.10) } \quad \Pi_{T} \mathcal{F}_{+}^{\text {loc }}:=L^{\infty}(0, T ; H) \cap L^{2}(0, T ; V) \cap\left\{u(\cdot) \mid \partial_{t} u(\cdot) \in L^{4 / 3}\left(0, T ; V^{\prime}\right)\right\} .
\end{aligned}
$$

The topology of space $\Pi_{T} \mathcal{F}_{+}^{\text {loc }}$ is defined as the following weak convergence: let $\left\{u_{n}(x, t)\right\}$ be a sequence of $\Pi_{T} \mathcal{F}_{+}^{\text {loc }}$, if

$$
\begin{cases}u_{n}(x, t) \rightarrow u(x, t) & \text { weak }^{*} \text { in } L^{\infty}(0, T ; H) \\ u_{n}(x, t) \rightarrow u(x, t) & \text { weakly in } L^{2}(0, T ; V) \\ \partial_{t} u_{n}(x, t) \rightarrow \partial_{t} u(x, t) & \text { weakly in } L^{4 / 3}\left(0, T ; V^{\prime}\right),\end{cases}
$$


then we say that the sequence of functions $\left\{u_{n}(x, t)\right\}$ converges to $u(x, t)$ in the topology of $\Pi_{T} \mathcal{F}_{+}^{\text {loc }}$ as $n \rightarrow \infty$.

The topology of space $\mathcal{F}_{+}^{\text {loc }}$ is the inductive limit of the topologies in the spaces $\Pi_{T} \mathcal{F}_{+}^{\text {loc }}$, i.e. a sequence of functions $\left\{u_{n}(x, t)\right\} \subset \mathcal{F}_{+}^{\text {loc }}$ converges to $u(x, t) \in \mathcal{F}_{+}^{\text {loc }}$ in the topology of $\mathcal{F}_{+}^{\text {loc }}$ as $n \rightarrow \infty$ if $\Pi_{T} u_{n}(x, t) \rightarrow \Pi_{T} u(x, t)$ in the topology of $\Pi_{T} \mathcal{F}_{+}^{\text {loc }}$ for any $T>0$. We denote by $\Theta_{+}^{\text {loc }}$ the space $\mathcal{F}_{+}^{\text {loc }}$ with this topology. We also define a Banach space $\mathcal{F}_{+}^{b}$ as

$$
\mathcal{F}_{+}^{b}:=\left\{u(x, t) \in \mathcal{F}_{+}^{\text {loc }} \mid\|u\|_{\mathcal{F}_{+}^{\mathrm{b}}}<+\infty\right\},
$$

where the norm in $\mathcal{F}_{+}^{b}$ is defined as

$$
\begin{aligned}
\|u\|_{\mathcal{F}_{+}^{b}}:=\sup _{t \geq 0}\left\{\|S(t) u\|_{L^{2}(0,1 ; V)}+\|S(t) u\|_{L^{\infty}(0,1 ; H)}\right. \\
\left.+\left\|S(t) \partial_{t} u\right\|_{L^{4 / 3}\left(0,1 ; V^{\prime}\right)}\right\} .
\end{aligned}
$$

Lemma 4.4 ([4]). Let $g \in V^{\prime}$. Then the translation semigroup $\{S(t)\}_{t \geq 0}$ defined by (3.1) possesses a trajectory attractor $\mathcal{A}_{0}^{\text {tr }} \subset \mathcal{T}_{0}^{+}$with respect to the topology $\Theta_{+}^{\text {loc }}$, which satisfies

(a) $\mathcal{A}_{0}^{\text {tr }}$ is bounded in $\mathcal{F}_{+}^{b}$ norm and is compact in the topology $\Theta_{+}^{\text {loc }}$;

(b) $S(t) \mathcal{A}_{0}^{\text {tr }}=\mathcal{A}_{0}^{\text {tr }}$, for all $t \geq 0$;

(c) $\mathcal{A}_{0}^{\text {tr }}$ is an attracting set for $\{S(t)\}_{t \geq 0}$ in the topology $\Theta_{+}^{\text {loc }}$, i.e. for any $\mathcal{B} \subset \mathcal{T}_{0}^{+}$bounded in $\mathcal{F}_{+}^{b}$ and for any neighbourhood $\mathcal{O}\left(\mathcal{A}_{0}^{\text {tr }}\right)$ of $\mathcal{A}_{0}^{\text {tr }}$, there is a time $t^{*}=t^{*}(\mathcal{B}, \mathcal{O})$ such that $S(t) \mathcal{B} \subset \mathcal{O}\left(\mathcal{A}_{0}^{\text {tr }}\right)$, for all $t \geq t^{*}$.

4.2. Convergence of solutions of the $3 \mathrm{D} \mathrm{CBF}$ equations to solutions of the 3D NS equations. In this subsection, we prove the convergence of solutions of the 3D CBF equations to solutions of the 3D NS equations as $\alpha \rightarrow 0^{+}$.

LEMmA 4.5. Let a sequence of functions $u_{\alpha_{n}}(t) \in \mathcal{T}_{\alpha_{n}}^{+}, n \in \mathbb{N}$, satisfy the following properties:

(a) $\left\{u_{\alpha_{n}}(\cdot)\right\}$ is uniformly (w.r.t. $n \in \mathbb{N}$ ) bounded in $\mathcal{F}_{+}^{b}$;

(b) $\alpha_{n} \rightarrow 0^{+}$as $n \rightarrow \infty$;

(c) $\left\{u_{\alpha_{n}}(\cdot)\right\} \rightarrow u(\cdot)$ in the topology $\Theta_{+}^{\text {loc }}$ as $n \rightarrow \infty$.

Then $u \in \mathcal{T}_{0}^{+}$.

Proof. By condition (a), (3.21), (3.23), (3.25), (3.27), (3.30) and (4.13), we see that there is some positive constant $C_{1}$ such that

$$
\left\|u_{\alpha_{n}}(\cdot)\right\|_{\mathcal{F}_{+}^{b}} \leq C_{1}, \quad\left\|u_{\alpha_{n}}(\cdot)\right\|_{\mathcal{F}_{\alpha,+}^{b}} \leq C_{1}, \quad \text { for all } n \in \mathbb{N} .
$$

Then (4.14), condition (c) and the lower semicontinuity of the norm give

$$
\|u(\cdot)\|_{\mathcal{F}_{+}^{b}} \leq C_{1} .
$$


We next prove that $u(\cdot)$ is a weak solution of the 3D NS equations on every $(0, T)$. Since $u_{\alpha_{n}}(\cdot) \in \mathcal{T}_{\alpha_{n}}^{+}$, the function $u_{\alpha_{n}}(\cdot)$ satisfies the equation

$$
\frac{\partial u_{\alpha_{n}}}{\partial t}+\nu \mathcal{L} u_{\alpha_{n}}+B\left(u_{\alpha_{n}}\right)+\alpha_{n} u_{\alpha_{n}}+\alpha_{n}\left|u_{\alpha_{n}}\right|^{r-1} u_{\alpha_{n}}=g
$$

in the sense $\mathcal{D}^{\prime}\left(0, T ; V^{\prime}\right)$. Clearly, by condition (c) and (4.11), we have

$$
\begin{array}{cc}
\frac{\partial u_{\alpha_{n}}}{\partial t} \rightarrow \frac{\partial u}{\partial t} & \text { weakly in } L^{4 / 3}\left(0, T ; V^{\prime}\right) \text { as } \alpha_{n} \rightarrow 0^{+}, \\
\alpha_{n} u_{\alpha_{n}} \rightarrow 0 & \text { weakly in } L^{2}(0, T ; V) \text { as } \alpha_{n} \rightarrow 0^{+} .
\end{array}
$$

Also, by the definition of the operator $\mathcal{L}$, we get

$$
\nu \mathcal{L} u_{\alpha_{n}} \rightarrow \nu \mathcal{L} u \quad \text { weakly in } L^{2}\left(0, T ; V^{\prime}\right) \text { as } \alpha_{n} \rightarrow 0^{+} .
$$

At the same time, by (3.25), (3.26), (3.30) and (4.14), we see that both $\left\{B\left(u_{\alpha_{n}}\right)\right\}$ and $\left\{\alpha_{n}\left|u_{\alpha_{n}}\right|^{r-1} u_{\alpha_{n}}\right\}$ are uniformly (w.r.t. $\left.n \in \mathbb{N}\right)$ bounded in $L^{4 / 3}\left(0, T ; V^{\prime}\right)$. Then by condition (c), it is not difficult to prove that

$$
\begin{aligned}
& B\left(u_{\alpha_{n}}\right) \rightarrow B(u) \text { weakly in } L^{4 / 3}\left(0, T ; V^{\prime}\right) \text { as } \alpha_{n} \rightarrow 0^{+}, \\
& \alpha_{n}\left|u_{\alpha_{n}}\right|^{r-1} u_{\alpha_{n}} \rightarrow 0 \quad \text { weakly in } L^{4 / 3}\left(0, T ; V^{\prime}\right) \text { as } \alpha_{n} \rightarrow 0^{+} \text {. }
\end{aligned}
$$

Since $L^{4 / 3}(0, T) \subset \mathcal{D}^{\prime}(0, T)$, we can pass the limit in (4.16) and obtain

$$
\frac{\partial u}{\partial t}+\nu \mathcal{L} u+B(u)=g, \quad \text { in } \mathcal{D}^{\prime}\left(0, T ; V^{\prime}\right),
$$

that is $u(\cdot)$ is a weak solution of the $3 \mathrm{D}$ NS equations on $(0, T)$.

We now prove that $u(\cdot)$ meets the energy inequality (4.8) for each $T>0$. From (4.14), we see that $\left\{\Pi_{T} u_{\alpha_{n}}\right\}$ is bounded in $L^{2}(0, T ; V)$ and $\left\{\Pi_{T} \partial_{t} u_{\alpha_{n}}\right\}$ is bounded in $L^{4 / 3}\left(0, T ; V^{\prime}\right)$. It then follows from Lemma 2.3 and condition (c) that

$$
\Pi_{T} u_{\alpha_{n}}(t) \rightarrow \Pi_{T} u(t) \quad \text { strongly in } L^{2}(0, T ; H) \text { as } \alpha_{n} \rightarrow 0^{+} .
$$

Thus, extracting a subsequence if necessary,

$$
\left\|\Pi_{T} u_{\alpha_{n}}(t)\right\|^{2} \rightarrow\left\|\Pi_{T} u(t)\right\|^{2} \text { for a.e. } t \in(0, T) \text { as } \alpha_{n} \rightarrow 0^{+} .
$$

For any $\psi(s) \in \mathcal{C}_{0}^{\infty}(0, T)$ with $\psi(s) \geq 0$, the sequence $\left\{\left\|\Pi_{T} u_{\alpha_{n}}(t)\right\|^{2} \psi^{\prime}(t)\right\}$ belongs to $L^{1}(0, T)$. From (4.14) we deduce that $\left\{\left\|\Pi_{T} u_{\alpha_{n}}(t)\right\|^{2} \psi^{\prime}(t)\right\}$ is essentially bounded and thus possesses an integrable majorant. Then by Lebesgue's dominated convergence theorem and (4.24), we have

$$
\int_{0}^{T}\left\|u_{\alpha_{n}}(s)\right\|^{2} \psi^{\prime}(s) d s \rightarrow \int_{0}^{T}\|u(s)\|^{2} \psi^{\prime}(s) d s \quad \text { as } \alpha_{n} \rightarrow 0^{+} .
$$

From condition (c), we have $u_{\alpha_{n}}(\cdot) \sqrt{\psi(\cdot)}-u(\cdot) \sqrt{\psi(\cdot)}$ weakly in $L^{2}(0, T ; V)$. Also by the lower semicontinuity of norm, we get

$$
\int_{0}^{T}\|u(s)\|^{2} \psi(s) d s \leq \liminf _{n \rightarrow \infty} \int_{0}^{T}\left\|u_{\alpha_{n}}(s)\right\|^{2} \psi(s) d s .
$$


Observing (4.23), we have

$$
\int_{0}^{T}\left\langle u_{\alpha_{n}}(s), g\right\rangle \psi(s) d s \rightarrow \int_{0}^{T}\langle u(s), g\rangle \psi(s) d s \quad \text { as } \alpha_{n} \rightarrow 0^{+} .
$$

Lastly, (4.14) implies that

$$
\int_{0}^{T}\left(\left\|u_{\alpha_{n}}(s)\right\|^{2}+\left\|u_{\alpha_{n}}(s)\right\|_{\widetilde{L}^{r+1}(\Omega)}^{r+1}\right) \psi(s) d s \leq C_{1}
$$

for any $n \in \mathbb{N}$. So we obtain

$$
\alpha_{n} \int_{0}^{T}\left(\left\|u_{\alpha_{n}}(s)\right\|^{2}+\left\|u_{\alpha_{n}}(s)\right\|_{\widetilde{\mathbb{L}}^{r+1}(\Omega)}^{r+1}\right) \psi(s) d s \rightarrow 0 \quad \text { as } \alpha_{n} \rightarrow 0^{+} .
$$

Since $u_{\alpha_{n}}(\cdot) \in \mathcal{T}_{\alpha_{n}}^{+}, n \in \mathbb{N}$, there holds

$$
\begin{aligned}
& -\frac{1}{2} \int_{0}^{T}\left\|u_{\alpha_{n}}(s)\right\|^{2} \psi^{\prime}(s) d s+\nu \int_{0}^{T}\left\|u_{\alpha_{n}}(s)\right\|_{V}^{2} \psi(s) d s \\
+ & \alpha \int_{0}^{T}\left(\left\|u_{\alpha_{n}}(s)\right\|^{2}+\left\|u_{\alpha_{n}}(s)\right\|_{\widetilde{\mathbb{L}}^{r+1}(\Omega)}^{r+1}\right) \psi(s) d s \leq \int_{0}^{T}\left\langle u_{\alpha_{n}}(s), g\right\rangle \psi(s) d s .
\end{aligned}
$$

Combining (4.25)-(4.28) and passing the limit in (4.29), we have

$$
-\frac{1}{2} \int_{0}^{T}\|u(s)\|^{2} \psi^{\prime}(s) d s+\nu \int_{0}^{T}\|u(s)\|_{V}^{2} \psi(s) d s \leq \int_{0}^{T}\langle u(s), g\rangle \psi(s) d s .
$$

(4.22) and (4.30) imply that $u \in \mathcal{T}_{0}^{+}$. The proof is complete.

4.3. Convergence of the trajectory attractor to that of the $3 \mathrm{D}$ NS equations. Here, we shall verify the main result of this section, that is, the trajectory attractor $\mathcal{A}_{\alpha}^{\mathrm{tr}}$ converges to the trajectory attractor $\mathcal{A}_{0}^{\mathrm{tr}}$ of the $3 \mathrm{D} \mathrm{NS}$ equations as $\alpha \rightarrow 0^{+}$.

Lemma 4.6. For each $\alpha \in(0,1]$, define

$$
\begin{aligned}
& \mathcal{B}_{\alpha}=\left\{u_{\alpha} \mid u_{\alpha} \in \mathcal{T}_{\alpha}^{+} \text {and there is some positive constant } C_{2}\right. \\
&\text { independent of } \left.\alpha \text { such that }\left\|u_{\alpha}\right\|_{\mathcal{F}_{+}^{b}} \leq C_{2} \text {, for all } u_{\alpha} \in \mathcal{B}_{\alpha}\right\} .
\end{aligned}
$$

Then

$$
S(t) \mathcal{B}_{\alpha} \rightarrow \mathcal{A}_{0}^{\mathrm{tr}} \quad \text { in the topology } \Theta_{+}^{\mathrm{loc}} \text { as } t \rightarrow+\infty \text { and } \alpha \rightarrow 0^{+},
$$

where (4.31) is interpreted in the following sense: for any sequence $\left\{u_{\alpha}\right\}_{0<\alpha \leq 1}$ with $u_{\alpha} \in \mathcal{B}_{\alpha}$, there is some $u \in \mathcal{A}_{0}^{\text {tr }}$ such that $S(t) u_{\alpha} \rightarrow u$ in the topology $\Theta_{+}^{\text {loc }}$ as $t \rightarrow+\infty$ and $\alpha \rightarrow 0^{+}$.

Proof. We argue by contradiction. Assume the converse: there exists some neighbourhood $\mathcal{O}\left(\mathcal{A}_{0}^{\text {tr }}\right)$ of $\mathcal{A}_{0}^{\text {tr }}$ in the topology $\Theta_{+}^{\text {loc }}$ and two sequences $\alpha_{n} \rightarrow 0^{+}$, $t_{n} \rightarrow+\infty$ as $n \rightarrow \infty$, such that

$$
S\left(t_{n}\right) \mathcal{B}_{\alpha_{n}} \not \subset \mathcal{O}\left(\mathcal{A}_{0}^{\text {tr }}\right)
$$


Then, there exist some solutions $u_{\alpha_{n}}(\cdot) \in \mathcal{B}_{\alpha_{n}}$ such that

$$
v_{\alpha_{n}}(t):=S\left(t_{n}\right) u_{\alpha_{n}}(t) \notin \mathcal{O}\left(\mathcal{A}_{0}^{\text {tr }}\right) .
$$

Since the function $v_{\alpha_{n}}(t)$ is a solution of the autonomous CBF equations (2.5)(2.6) with $\alpha=\alpha_{n}$ on the interval $\left[-t_{n},+\infty\right)$ and $v_{\alpha_{n}}(t)$ is the backward time shift of the solution $u_{\alpha_{n}}(t)$ by $t_{n}$, we get from the definition of $\mathcal{B}_{\alpha}$ that

$$
\begin{aligned}
\sup _{t \geq-t_{n}}\left\|v_{\alpha_{n}}(t)\right\|+\sup _{t \geq-t_{n}} & \left(\int_{t}^{t+1}\left\|v_{\alpha_{n}}(s)\right\|_{V}^{2} d s\right)^{1 / 2} \\
& +\sup _{t \geq-t_{n}}\left(\int_{t}^{t+1}\left\|\partial_{t} v_{\alpha_{n}}(s)\right\|_{V^{\prime}}^{4 / 3} d s\right)^{3 / 4} \leq C_{2} .
\end{aligned}
$$

For each $T>0$, we consider $\alpha_{n}$ with the index $n$ such that $t_{n} \geq T$. Then (4.34) implies that the sequence $\left\{v_{\alpha_{n}}(\cdot)\right\}$ is weakly compact in the space

$$
\Theta_{-T, T}:=L^{\infty}(-T, T ; H) \cap L^{2}(-T, T ; V) \cap\left\{v \mid \partial_{t} v \in L^{4 / 3}\left(-T, T ; V^{\prime}\right)\right\}
$$

for every fixed $T>0$, where the topology of $\Theta_{-T, T}$ is defined in the manner similar to $(4.11)$ with the interval $(0, T)$ replaced by $(-T, T)$. Hence, for every fixed $T>0$, we can choose a subsequence $\left\{n_{k}\right\}$ such that $\left\{v_{n_{k}}(\cdot)\right\}$ converges weakly in $\Theta_{-T, T}$. Then, using the standard diagonal procedure, we can construct a function $v(t), t \in \mathbb{R}$, and a subsequence $\left\{n_{k_{j}}\right\}$ such that

$$
v_{n_{k_{j}}}(\cdot) \rightarrow v(\cdot) \quad \text { weakly in } \Theta_{-T, T} \text { as } n_{k_{j}} \rightarrow \infty \text {, for each } T>0 .
$$

So, by (4.34), we obtain the following inequality for the limit function $v(t)$

$$
\begin{aligned}
\sup _{t \in \mathbb{R}}\|v(t)\|+\sup _{t \in \mathbb{R}}\left(\int_{t}^{t+1}\|v(s)\|_{V}^{2} d s\right)^{1 / 2} & \\
& \left.+\sup _{t \in \mathbb{R}}\left(\int_{t}^{t+1} \| \partial_{t} v(s)\right) \|_{V^{\prime}}^{4 / 3} d s\right)^{3 / 4} \leq C_{2} .
\end{aligned}
$$

Thus, we get $v \in \mathcal{F}^{b}:=\left\{u \in \mathcal{F}^{\text {loc }} \mid\|u\|_{\mathcal{F}^{\mathrm{b}}}<+\infty\right\}$, where

$$
\mathcal{F}^{\text {loc }}:=L^{\infty}(\mathbb{R} ; H) \cap L_{\text {loc }}^{2}(\mathbb{R} ; V) \cap\left\{u(\cdot) \mid \partial_{t} u(\cdot) \in L_{\text {loc }}^{4 / 3}\left(\mathbb{R} ; V^{\prime}\right)\right\}
$$

and the norm in $\mathcal{F}^{b}$ is defined as

$$
\begin{aligned}
\|v\|_{\mathcal{F}^{b}}:=\sup _{t \in \mathbb{R}}\left\{\|S(t) u\|_{L^{2}(0,1 ; V)}\right. & \\
& \left.+\|S(t) u\|_{L^{\infty}(0,1 ; H)}+\left\|S(t) \partial_{t} u\right\|_{L^{4 / 3}\left(0,1 ; V^{\prime}\right)}\right\} .
\end{aligned}
$$

Since the addressed equation is autonomous, we can apply Lemma 4.5 in which we suppose that all the functions are defined on the semi-axis $[-T,+\infty)$ instead of $[0,+\infty)$. By doing so and using (4.35)-(4.36), we see that $v(x, t)$ is a weak solution of the 3D NS equations for all $t \in \mathbb{R}$. This fact, together with (4.36), implies $v \in \mathcal{K}_{0}$, where $\mathcal{K}_{0}$ is the kernel of equation (4.5). By the structure of 
the trajectory attractor (see [4]), we have $\Pi_{+} \mathcal{K}_{0}=\mathcal{A}_{0}^{\text {tr }}$. Then we conclude that $\Pi_{+} v \in \Pi_{+} \mathcal{K}_{0}=\mathcal{A}_{0}^{\text {tr }}$ and by $(4.35)$

$$
\Pi_{+} v_{n_{k_{j}}} \rightarrow \Pi_{+} v \text { in the topology } \Theta_{+}^{\text {loc }} \text { as } n \rightarrow \infty .
$$

Particularly, we have for large enough $n_{k_{j}}$ that

$$
\Pi_{+} v_{n_{k_{j}}} \in \mathcal{O}\left(\Pi_{+} v\right) \subset \mathcal{O}\left(\mathcal{A}_{0}^{\mathrm{tr}}\right),
$$

which contradicts (4.32). The proof is complete.

TheOREM 4.7. Let $\mathcal{A}_{\alpha}^{\text {tr }}$ and $\mathcal{A}_{0}^{\text {tr }}$ be the trajectory attractor for the CBF equation (2.5) and the 3D NS equation (4.5), respectively. Then

$$
\mathcal{A}_{\alpha}^{\text {tr }} \rightarrow \mathcal{A}_{0}^{\text {tr }} \quad \text { in the topology } \Theta_{+}^{\text {loc }} \quad \text { as } \alpha \rightarrow 0^{+} .
$$

Proof. We see from Section 3 that the family $\left\{\mathcal{A}_{\alpha}^{\text {tr }}\right\}_{0<\alpha \leq 1}$ is uniformly (w.r.t. $\alpha \in(0,1])$ bounded in $\mathcal{F}_{+}^{b}$ norm. Applying Lemma 4.6 with $\mathcal{B}_{\alpha}=\mathcal{A}_{\alpha}^{\text {tr }}$ and the invariant property of $\mathcal{A}_{\alpha}^{\text {tr }}$ (see Theorem 3.9(c)), we obtain

$$
\mathcal{A}_{\alpha}^{\text {tr }}=S(t) \mathcal{A}_{\alpha}^{\text {tr }} \rightarrow \mathcal{A}_{0}^{\text {tr }} \quad \text { in the topology } \Theta_{+}^{\text {loc }} \text { as } t \rightarrow+\infty \text { and } \alpha \rightarrow 0^{+} .
$$

The proof is complete.

Acknowledgements. We warmly thank the anonymous referee for his/her pertinent comments and suggestions, which greatly improved the earlier manuscript.

\section{REFERENCES}

[1] R.A. Adams, Sobolev Spaces, Academic Press, New York, 1975.

[2] J.M. BALL, Continuity properties of global attractors of generalized semiflows and the Navier-Stokes equations, J. Nonlinear Sci. 7 (1997), 475-502.

[3] V.V. Chepyzhov And M.I. Vishik, Evolution equations and their trajectory attractors, J. Math. Pures Appl. 76 (1997), 913-664.

[4] - Attractors for Equations of Mathematical Physics, AMS, R.I., 2002.

[5] V.V. Chepyzhov, E.S. Titi And M.I. Vishik, On the convergence of solutions of the Leray $-\alpha$ model to the trajectory attractor of the 3D Navier-Stokes system, Discrete Contin. Dyn. Syst. (A) 17 (2007), 481-500.

[6] T. Caraballo, J. Langa and J. Valero, Global attractors for multivalued random dynamical systems generated by random differential inclusions with multiplicative noise, J. Math. Anal. Appl. 260 (2001), 602-622.

[7] _ Global attractors for multivalued random semiflows generated by random differential inclusions with additive noise, C.R. Acad. Sci. Paris Sér. I 331 (2001), 131-136.

[8] _ Global attractors for multivalued random dynamical systems, Nonlinear Anal. 48 (2002), 805-829.

[9] T. Caraballo, J. Langa, V. Melnik and J. Valero, Pullback attractors of nonautonomous and stochastic multivalued dynamical systems, Set-Valued Anal. 11 (2003), $153-201$

[10] T. Caraballo, P.E. Kloeden and P.M. Rubio, Weak pullback attractors of setvalued processes, J. Math. Anal. Appl. 288 (2003), 692-707. 
[11] O. Çelebi, V. Kalantarov and D. UĞUrlu, On continuous dependence on coefficients of the Brinkman-Forchheimer equations, Appl. Math. Lett. 19 (2006), 801-807.

[12] M. Firdaouss, J.-L. Guermond and P. Le Quéré, Nonlinear corrections to Darcy's law at low Reynolds numbers, J. Fluid Mechanics 343 (1997), 331-350.

[13] F. Franchi, B. Straughan, Continuous dependence and decay for the Forchheimer equations, R. Soc. Lond. Proc. Ser. A: Math. Phys. Eng. Sci. 459 (2003), 3195-3202.

[14] T. Giongi, Derivation of the Forchheimer law via matched asymptotic expansions, Transl. Porous Media 29 (1997), 191-206.

[15] A. Kapustyan and J. Valero, Weak and strong attractors for the 3D Navier-Stokes system, J. Differential Equations 240 (2007), 249-278.

[16] V.K. Kalantarov And S. ZELIK, Smooth attractor for the Brinkman-Forchheimer equations with fast growing nonlinearities, Comm. Pure Appl. Anal. 5 (2012), 2037-2054.

[17] V. Melnik and J. Valero, On attractors of multivalued semi-flows and differential inclusions, Set-Valued Anal. 6 (1998), 83-111.

[18] _ On global attractors of multivalued semiprocess and nonautonomous evolution inclusions, Set-Valued Anal. 8 (2000), 375-403.

[19] Y. OuYang And L. Yan, A note on the existence of a global attractor for the BrinkmanForchheimer equations, Nonlinear Anal., 70 (2009), 2054-2059.

[20] L.E. Payne, J.C. Song and B. Straugham, Continuous dependence and convergence results for Brinkman and Forchheimer models with variable viscosity, R. Soc. Lond. Proc. Ser. A: Math. Phys. Eng. Sci. 455 (1999), 2173-2190.

[21] L.E. Payne and B. Straugham, Convergence and continuous dependence for the Brinkman-Forchheimer equations, Studies Appl. Math., 102 (1999), 419-439.

[22] A. Shenoy, Non-Newtonian fluid heat transfer in porous media, Adv. Heat Transfer 24 (1994), 101-190.

[23] B. Straughan, Stability and Wave Motion in Porous Media, Springer, New York, 2008.

[24] R. Temam, Navier-Stokes Equations, Theory and Numerical Analysis, North-Holland, Amsterdam, 1984.

[25] — Infinite Dimensional Dynamical Systems in Mechanics and Physics, Springer, Berlin, 2nd ed., 1997.

[26] D. UĞURLU, On the existence of a global attractor for the Brinkman-Forchheimer equations, Nonlinear Anal. 68 (2008), 1986-1992.

[27] M.I. VishiK AND V.V. ChePyzhov, Trajectory and global attractors of 3D Navier-Stokes systems, Math. Notes, 77 (2002), 177-193.

[28] _ , Trajectory attractors of equations of mathematical physics, Russian Math. Surveys 4 (2011), 639-731.

[29] M.I. Vishik, E.S. Titi And V.V. Chepyzhov, On the convergence of trajectory attractor of the 3D Navier-Stokes- $\alpha$ model as $\alpha$ approaches 0, Sb. Math. 198 (2007), 1703-1736.

[30] B. WANG AND S. Lin, Existence of global attractors for the three-dimensional BrinkmanForchheimer equation, Math. Meth. Appl. Sci. 31 (2008), 1479-1495.

[31] Y. WANG AND S. ZHOU, Kernel sections and uniform attractors of multi-valued semiprocesses, J. Differential Equations 232 (2007), 573-622.

[32] S. Whitaker, The Forchheimer equation: A theoretical development, Transl. Porous Media 25 (1996), 27-62.

[33] Y. You, C. Zhao and S. Zhou, The existence of uniform attractors for 3D BrinkmanForhheimer equations, Discrete Contin. Dyn. Syst. (A) 3 (2012), 3787-3800. 
[34] C. ZHAO AND Y. You, Approximation of the incompressible convective BrinkmanForchheimer equations, J. Evolution Equations 12 (2012), 767-788.

Caidi Zhao, Lei Kong and Guowei LiU

Department of Mathematics and Information Science

Wenzhou University

325035 Wenzhou, ZheJiang Province, P.R. CHINA

E-mail address: zhaocaidi2013@163.com

Min ZhaO

College of Life and Environmental Science

Wenzhou University

325035 Wenzhou, ZheJiang Province, P.R. CHINA

TMNA : VOLUME $44-2014-\mathrm{N}^{\mathrm{O}} 2$ 